\title{
The cardioprotective effect of microemulsion propofol against ischemia and reperfusion injury in isolated rat heart
}

\author{
Min Jung Hur, Heezoo Kim, Dong Kyu Lee, and Sang Ho Lim \\ Department of Anesthesiology and Pain Medicine, Korea University Medical Center, Guro Hospital, Seoul, Korea
}

Background: Lipid-emulsion propofol (LP) has cardioprotective effects against ischemia-reperfusion injury, but it has lipid-related side effects. Microemulsion propofol (MP) is a lipid-free propofol emulsified with $10 \%$ purified poloxamer 188 (PP188). PP188 is a nonionic surfactant and has cardioprotective effects. However, some reports have suggested that reduced cardioprotective effects were observed when the cardioprotective agents were used in combination even though each cardioprotective agent has cardioprotective effects. The aims of this study were to examine and compare the cardioprotective effects of MP and LP.

Methods: 50 isolated rat hearts were perfused with modified Kreb's solution. They were divided into 4 groups and underwent 30 minutes of ischemia and 60 minutes of reperfusion. Control group: ischemia-reperfusion was performed without treatment. LP, MP and PP groups: LP, MP and PP188 were infused during the pre-ischemic and reperfusion period, respectively. Hemodynamic parameters and coronary effluent flow rate (CEFR) were measured. Infarct size was determined using triphenyl-tetrazolium staining.

Results: In the MP group, systolic pressure was maintained near baseline, the systolic pressure was higher than that in the other groups and HR was lower than that in the other groups during reperfusion. Diastolic pressure was transiently increased in the PP group after treatment and at 5 minutes after reperfusion compared with that in the control group and in the the LP group. There were no differences in $\mathrm{dP} / \mathrm{dt}_{\max }$ and CEFR between groups. Infarct size in the LP, MP and PP groups was smaller than that in the control group, but there were no significant differences between these three groups.

Conclusions: MP has cardioprotective effects similar to those of LP. MP can be used for cardiac anesthesia in cases with ischemia-reperfusion injury to avoid the lipid-related side effects of LP. (Korean J Anesthesiol 2012; 62: 358-364)

Key Words: Heart, Ischemia, Microemulsion propofol, Reperfusion injury.

Received: November 22, 2011. Revised: 1st, January 6, 2012; 2nd, January 18, 2012. Accepted: January 19, 2012.

Corresponding author: Sang Ho Lim, M.D., Ph.D., Deptartment of Anesthesiology and Pain Medicine, Korea University Medical Center, Guro Hospital, Guro 2-dong, Guro-gu, Seoul 152-703, Korea. Tel: 82-2-2626-1437, Fax: 82-2-851-9897, E-mail: lsh1102@unitel.co.kr

(c) This is an open-access article distributed under the terms of the Creative Commons Attribution Non-Commercial License (http:// creativecommons.org/licenses/by-nc/3.0/), which permits unrestricted non-commercial use, distribution, and reproduction in any medium, provided the original work is properly cited. 


\section{Introduction}

Extracorporeal circulation is required in cardiac transplantation or in open heart surgery during which the heart undergoes artificial ischemia and reperfusion, which play an important role in reperfusion injuries including reversible myocardial stunning and irreversible damage such as myocardial infarction. Although most of the general anesthetic drugs have negative inotropic effects, there are agents that have a cardioprotective effect in myocardial ischemia and reperfusion injury [1]. Particularly, propofol has been reported to have cardioprotective effects through reduction of free oxygen radicals [2] and calcium overload phenomenon [3]. In addition, propofol allows hemodynamic stability during anesthesia, and it has been widely used in cardiac surgery and for postoperative sedation in the intensive care unit. However, several lipid-associated problems such as hypertriglyceridemia, pulmonary embolism and extrinsic contamination due to rapid bacterial growth at room temperature, have been reported with the long-term use of lipid-emulsion propofol (LP: Diprivan ${ }^{\circledR}$, AstraZeneca, London, UK) [4-6]. To eliminate these adverse effects of lipid-emulsion propofol, microemulsion propofol (MP: Aquafol ${ }^{\circledR}$, Daewon Pharmaceutical Co., Ltd., Seoul, Korea) emulsified with hydrophilic polymer (10\% purified poloxamer 188) was developed. Poloxamer 188 (PP188) is nontoxic surface-active agent that has cytoprotective effects. PP188 is incorporated into the lipid bilayers, thereby decreasing their susceptibility to oxidative stress and inflammation [711]. Inhaled anesthetics, propofol, and PP188 provide cardioprotection against ischemia and reperfusion injury via different mechanisms [11], but despite these distinct mechanisms several studies have demonstrated that no additive or superior protection was observed with the combination of these cardioprotective agents [12,13].

Previous studies mainly focused on pharmacokinetic and pharmacodynamic characteristics of MP, and MP demonstrated pharmacokinetic and pharmacodynamic properties similar to those of LP [14]. However, the difference between LP and MP in terms of myocardial protection against ischemia and reperfusion injury during cardiac surgery is unknown.

The purpose of this study was to compare the myocardial protective effects between LP and MP emulsified with PP188 by measuring hemodynamic parameters and infarct size in Langendorff perfused rat hearts.

\section{Materials and Methods}

This experimental study was approved by Institutional Animal Care and Use Committee. Male Sprague-Dawley rats $(250-350 \mathrm{~g})$ were allowed access to food and water until the day of surgery. Rats were anesthesized with pentobarbital (25$30 \mathrm{mg}$, intraperitoneally). After confirming the loss of light reflex and responsiveness, rats were heparinized with sodium heparin (250 IU, intraperitoneally) and were intubated with a $16 \mathrm{G}$ catheter via tracheostomy. Ventilation was maintained with animal ventilator (SN-480-7, Shinano Co., Nagano, Japan) with mixed gas (oxygen $95 \%$, carbon dioxide $5 \%$ ) at a respiratory rate of 50 breaths per minutes and a tidal volume of 3-4 ml. After median sternotomy, heart was rapidly excised en bloc and then was immersed in ice-cold perfusion solution to prevent myocardial injury during the remaining procedure. All hearts (coronary arteries) were perfused retrogradely via the aorta on the Langendorff apparatus with modified Kreb's solution containing $118 \mathrm{mM} \mathrm{NaCl}, 4.7 \mathrm{mM} \mathrm{KCl}, 1.2 \mathrm{mM} \mathrm{MgSO}_{4}, 1.2 \mathrm{mM}$ $\mathrm{KH}_{2} \mathrm{PO}_{4}, 25 \mathrm{mM} \mathrm{NaHCO}, 10 \mathrm{mM}$ glucose, $1.9 \mathrm{mM} \mathrm{CaCl}_{2}$, and $0.5 \mathrm{mM}$ Na-EDTA. The perfusate solution was maintained at $37 \pm 0.2^{\circ} \mathrm{C}$ using a thermostatically controlled water circulating system (GP-200, NESLAB Instrument Inc., USA) and was kept at $\mathrm{pH} 7.4 \pm 0.02$ with $3 \mathrm{~L} / \mathrm{min}$ mixed gas (oxygen $95 \%$, carbon dioxide $5 \%$ ). Perfusion pressure was maintained at $55 \mathrm{mmHg}$ by a 75-cm high fluid column with an overflow pump (Masterflex, Cole-Parmer Instrument Co., USA), and was measured at the level of the aortic root via a pressure transducer. A $3 \mathrm{~mm}$ saline-filled latex balloon connected to the pressure transducer (Ohmeda P23XL transducer, Ohmeda, Oxnard, CA, USA) was inserted into the left ventricle through the mitral valve via a left atrial incision and balloon volume was adjusted to maintain a diastolic pressure of $10 \mathrm{mmHg}$ after equilibration of the heart.

All hearts were perfused on Langendorff apparatus and were stabilized for about 30 minutes in order to obtain the baseline measurements. Each group received a sequential experimental protocol including pretreatment, global ischemia and reperfusion. After stabilization, pretreatment was performed for 15

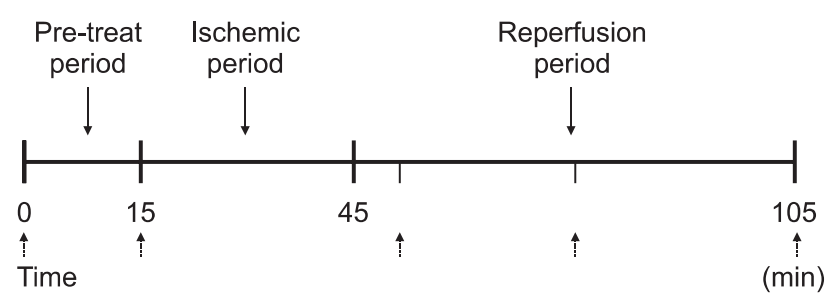

Fig. 1. Experimental protocol. After stabilization, each group was subjected to a 15 minutes pre-treat period, 30 minutes of global ischemia and 60 minutes of reperfusion. Specific agents were applied to each group as follows: perfusate and mixed gas $\left(95 \% \mathrm{O}_{2}+5 \% \mathrm{CO}_{2}\right)$ only for control group; mixed gas with perfusate containing $6 \mu \mathrm{g} /$ $\mathrm{ml}$ lipid-emulsion propofol for LP group; $6 \mu \mathrm{g} / \mathrm{ml}$ microemulsion propofol for MP group; $60 \mu \mathrm{g} / \mathrm{ml}$ poloxamer 188 for PP group. Hemodyamic variables were measured at after stabilization, after pre-treat period and 5, 30, 60 minutes after reperfusion started (Dashed arrows). After all processes were completed, triphenyl tetrazolium staining was performed to calculate infarct size. 
minutes in each group (as described below). By blocking the perfusion towards the hearts, global ischemia was induced for 30 minutes and this was then followed by restarting the perfusion for 60 minutes. Experiments were conducted in the 4 groups, the control group underwent pretreatment and reperfusion without any medication, and the LP, MP and PP groups were treated with LP, MP and PP188 (Lutrol ${ }^{\circledR}$ F68, Sigma-Aldrich Korea Ltd., Kyunggi-Do, Korea), respectively during the pretreatment time and reperfusion. Each group was placed in a random order by using block randomization (Fig. 1).

Using the pressure sensor, hemodynamic parameters were measured after stabilization (baseline), before ischemia (after pretreatment), and at 5, 30 and 60 minutes after reperfusion. These parameters were automatically stored in the computer using MP-100 system (Biopac ${ }^{\circledR}$ System Inc., Goleta, CA, USA) and Acknowledge software (MP 100 manager version 3.8.2, Biopac $^{\circledR}$ system Inc., Goleta, CA, USA). A cannula was inserted into the coronary sinus to measure the coronary effluent flow rate. Coronary effluent flow rate was defined as the flow per minute at each measurement point.

Perfusion fluid containing LP and MP corresponding to $6 \mu \mathrm{g} / \mathrm{ml}$ of propofol solution were used during pretreatment and reperfusion. Because MP contains 10\% PP188, PP188 was diluted in the same way as MP which corresponded to $60 \mu \mathrm{g} / \mathrm{ml}$ of PP188 in the perfusion fluid.

After reperfusion, the hearts were perfused with cold cardio-

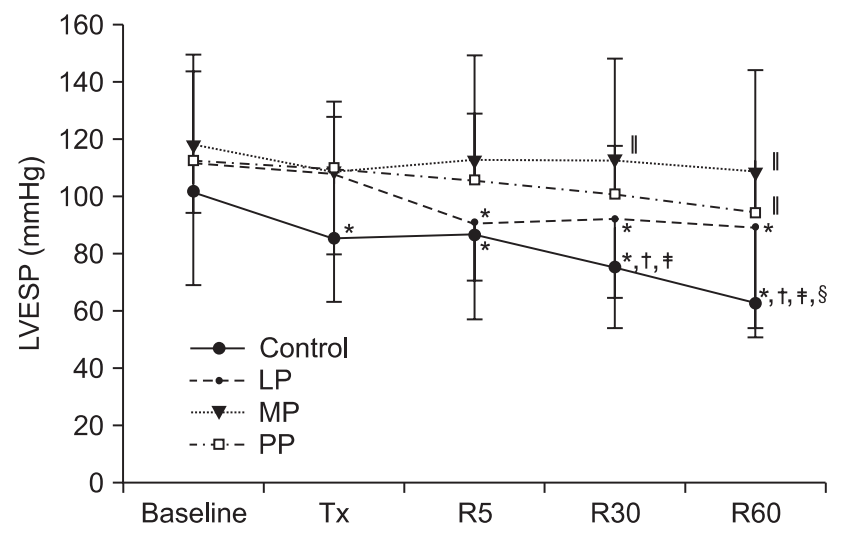

Fig. 2. The changes in the left ventricular end systolic pressure (LVESP). Values are expressed as mean \pm SD. Control group: no treatment during pre-ischemic and reperfusion period, LP, MP and PP groups: lipid emulsion propofol, microemulsion propofol and PP188 were infused during pre-ischemic and reperfusion period, respectively. Baseline: immediately after stabilization, Tx: preischemic treatment, R5: at 5 minutes after reperfusion, R30: at 30 minutes after reperfusion, R60: at 60 minutes after reperfusion. Probability value was indicated as follows; $* \mathrm{P}<0.05$ vs. baseline, ${ }^{\dagger}$ vs. Tx, ${ }^{\ddagger}$ vs. R5, ${ }^{\S}$ vs. R30, "vs. control group, "vs. LP group, ** vs. MP group. In the control group and in the LP group, LVESP significantly decreased during the reperfusion period compared with baseline, however in the MP group there was no significant decrease in LVESP. plegic solution and immersed in ice-cold perfusate to achieve cardiac arrest. Then the hearts were immediately frozen to $-20^{\circ} \mathrm{C}$. Whole ventricular muscles were sliced at $2 \mathrm{~mm}$ intervals and stained with triphenyl tetrazolium. The slices were incubated for 10 minutes in $1 \%$ solution of buffered triphenyl tetrazolium (2, 3, 5-triphenyl tetrazolium chloride: $\mathrm{C}_{19} \mathrm{H}_{15} \mathrm{ClN}_{4}$, TTC) at $37^{\circ} \mathrm{C}$ and then immersed in $10 \%$ buffered formaldehyde for 20 minutes. The stained slices were then placed between two sheets of glass with $1.7 \mathrm{~mm}$ shims at the corners for squashing the slices to a uniform thickness, and the size of the damaged and the whole part of the ventricular muscles were measured using UTHSCSA Image Tool, version 3.0 (S. Brent Dove, University of Texas Health Science Center in San Antonio, Texas, USA). Then the heart volume of the damaged heart and the entire heart volume were calculated and infarct size was determined as the ratio (\%) of the heart volume of the damaged heart to the entire heart volume.

A total of 50 hearts were prepared for this experiment. Among these, 6 extracted hearts were excluded due to a surgical error or inappropriate cardiac function until stabilization. Finally, 44 hearts were included in the statistical analysis; the control group contained 14 hearts, the LP, MP and PP groups contained 10 hearts, respectively.

All statistics were calculated using SigmaPlot for Windows version 12.0 (Systat Software, Inc., IL, USA). Data were subjected to one-way analysis of variance (ANOVA) and one-way repeated measures ANOVA, and if statistical significance was established,

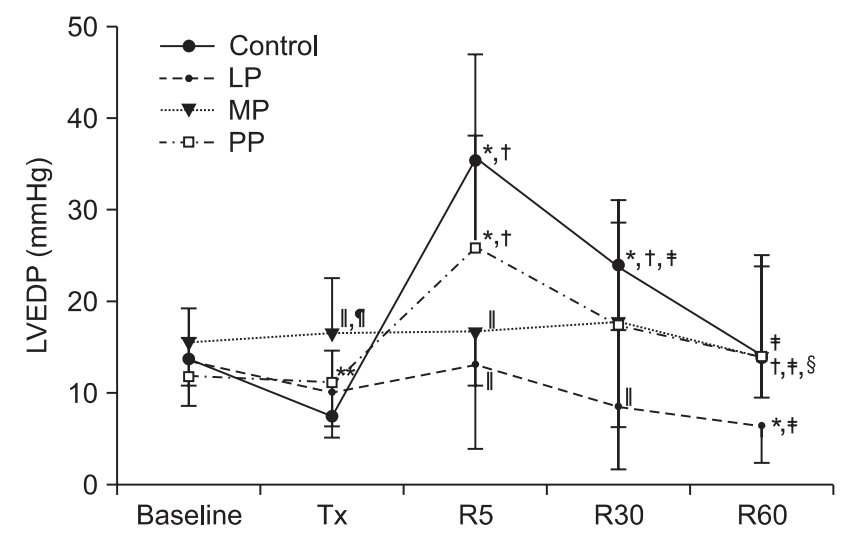

Fig. 3. The changes in the left ventricular end diastolic pressure (LVEDP). Values are expressed as mean \pm SD. Control group: no treatment during pre-ischemic and reperfusion period, LP, MP and PP groups: lipid emulsion propofol, microemulsion propofol and PP188 were infused during pre-ischemic and reperfusion period, respectively. Baseline: immediately after stabilization, Tx: preischemic treatment, R5: at 5 minutes after reperfusion, R30: at 30 minutes after reperfusion, R60: at 60 minutes after reperfusion. Probability value was indicated as follows; ${ }^{*} \mathrm{P}<0.05$ vs. baseline, ${ }^{\dagger}$ vs. Tx, ${ }^{\ddagger}$ vs. R5, ${ }^{\S}$ vs. R30, "vs. control group, "vs. LP group, **vs. MP group. LVEDP was significantly higher in the MP group than in the control group and in the LP group. At R5, in the PP group, LVEDP was significantly increased than that at baseline and at Tx. 
values of the control group and those of the other groups were compared using Bonferroni t-test. For all studies, statistical significance was considered at $\mathrm{P}<0.05$.

\section{Results}

\section{Left ventricular end systolic pressure (LVESP, Fig. 2)}

In the control group and in the LP group, LVESP was decreased after 5 minutes, 30 minutes and 60 minutes of reperfusion and the values were significantly lower compared to the baseline value (Control group: $\mathrm{P}<0.001$, LP group: $\mathrm{P}=0.001$ ). In contrast, the MP group did not show significant changes in LVESP $(\mathrm{P}=0.307)$ and in the PP group LVESP was decreased after reperfusion compared to the baseline value but the difference was not statistically significant $(\mathrm{P}=0.070)$. After 30 and 60 minutes of reperfusion, LVESP was significantly higher in the MP group and in the PP group than in the control group (at 40 minutes of reperfusion: $\mathrm{P}=0.009$, at 60 minutes of reperfusion: $\mathrm{P}=$ 0.001).

\section{Left ventricular end diastolic pressure (LVEDP, Fig. 3)}

At 5 minutes of reperfusion, LVEDP was increased in the control group and in the PP group compared to the baseline value and LVEDP progressively decreased at 30 and 60 minutes

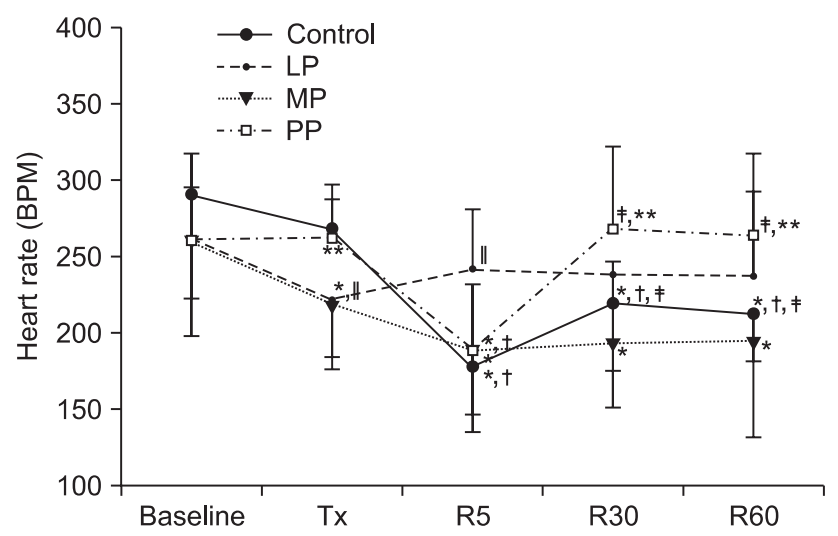

Fig. 4. The changes in heart rate (HR). Values are expressed as mean \pm SD. Control group: no treatment during pre-ischemic and reperfusion period, LP group: lipid emulsion propofol was infused during pre-ischemic and reperfusion period, MP group: microemulsion propofol was infused during pre-ischemic and reperfusion period, PP group: PP188 was infused during preischemic and reperfusion period. Baseline: immediately after stabilization, Tx: pre-ischemic treatment, R5: at 5 minutes after reperfusion, R30: at 30 minutes after reperfusion, R60: at 60 minutes after reperfusion. Probability value was indicated as follows; $* \mathrm{P}<$ 0.05 vs. baseline, ${ }^{\dagger}$ vs. Tx, ${ }^{\dagger}$ vs. R5, ${ }^{\S}$ vs. R30, "vs. control group, "vs. LP group, **vs. MP group. In the MP group, HR was significantly reduced after reperfusion compared with that at the baseline. of reperfusion $(\mathrm{P}<0.001)$. In the LP group, LVEDP after reperfusion was maintained near baseline, and at 60 minutes of reperfusion, LVEDP was significantly decreased compared to the baseline value $(\mathrm{P}=0.008)$. In the MP group also, LVEDP was maintained near baseline $(\mathrm{P}=0.428)$. In the LP group and in the MP group, LVEDP was lower than that in the control group at 5 minutes of reperfusion $(\mathrm{P}<0.001)$ and in the LP group, the LVEDP value was lower than that in the control group even at 30 minutes of reperfusion $(\mathrm{P}=0.003)$.

\section{Heart rate (HR, Fig. 4)}

In the LP group, HR was decreased after pretreatment, but after reperfusion it was maintained near baseline $(\mathrm{P}=0.032)$. In the MP group, HR was decreased after pretreatment and low values were continued during reperfusion $(\mathrm{P}<0.001)$. In the $\mathrm{PP}$ group, HR was decreased only at 5 minutes of reperfusion $(\mathrm{P}$ $=0.002$ ), and the HR values were higher than those in the MP group after pretreatment, at 30 and 60 minutes after reperfusion $(\mathrm{P}=0.001, \mathrm{P}<0.001$ and $\mathrm{P}=0.021$, respectively).

\section{Left ventricular pressure development over time (dP/dt max $_{\text {Fig. 5) }}$}

At $5 \mathrm{~min}$ of reperfusion, $\mathrm{dP} / \mathrm{dt}_{\max }$ was significantly reduced than the baseline values of all 4 groups (Control group, PP188

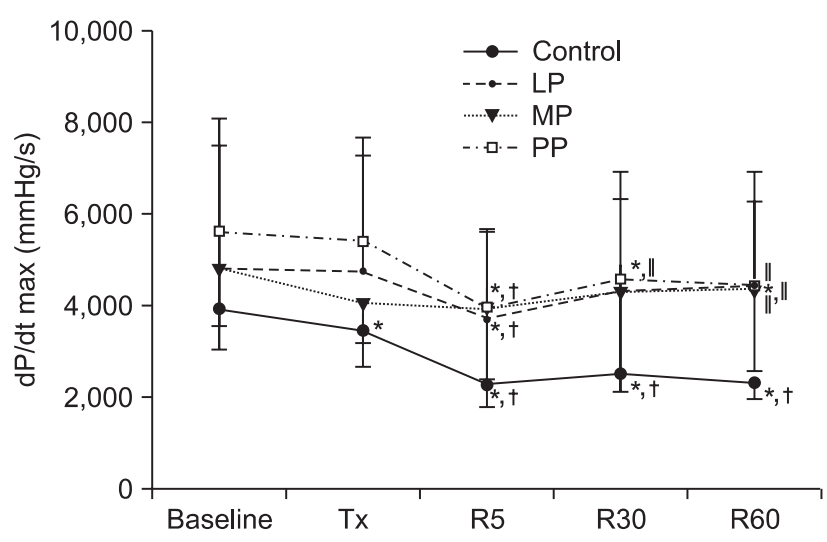

Fig. 5. The changes in the maximum rate of change in left ventricular pressure $\left(\mathrm{dP} / \mathrm{dt}_{\max }\right)$. Values are expressed as mean $\pm \mathrm{SD}$. Control group: no treatment during pre-ischemic and reperfusion period, LP group: lipid emulsion propofol was infused during pre-ischemic and reperfusion period, MP group: microemulsion propofol was infused during pre-ischemic and reperfusion period, PP group: PP188 was infused during pre-ischemic and reperfusion period. Baseline: immediately after stabilization, Tx: pre-ischemic treatment, R5: at 5 minutes after reperfusion, R30: at 30 minutes after reperfusion, R60: at 60 minutes after reperfusion. Probability value was indicated as follows; *P $<0.05$ vs. baseline, ${ }^{\dagger}$ vs. Tx, ${ }^{\ddagger}$ vs. R5, ${ }^{\S}$ vs. R30, "vs. control group, "vs. LP group, **vs. MP group. All groups demonstrated decreased values after reperfusion but there was no significant difference in $\mathrm{dP} / \mathrm{dtmax}$ among the LP, MP and PP groups. 
group: $\mathrm{P}<0.001$, LP group: $\mathrm{P}=0004$, MP group: $\mathrm{P}=0.006)$. In the LP and MP groups, $\mathrm{dP} / \mathrm{dt}_{\max }$ gradually recovered after 5 minutes of reperfusion. At 5, 30 and 60 minutes of reperfusion, $\mathrm{dP} / \mathrm{dt}_{\max }$ was significantly decreased in the PP group compared to the baseline values $(\mathrm{P}<0.001)$. At 30 minutes of reperfusion, $\mathrm{dP} / \mathrm{dt}_{\max }$ was significantly higher in the PP group than in the control group, and at 60 minutes of reperfusion, $\mathrm{dP} / \mathrm{dt}_{\max }$ was significantly higher in the LP, MP and PP groups than in the control group (at 30 minutes of reperfusion: $\mathrm{P}=0.020$, at 60 minutes of reperfusion: $\mathrm{P}=0.006$ ).

\section{Coronary effluent flow rate (CEFR, Fig. 6)}

In the LP group, CEFR gradually decreased after reperfusion, the values at 30 and 60 minutes of reperfusion were lower than the baseline and/or after pretreatment values $(\mathrm{P}=0.003)$. During reperfusion periods, CEFR was higher in the LP group than in the control group (at 5 minutes of reperfusion: $\mathrm{P}<$ 0.001 , at 30 minutes of reperfusion: $\mathrm{P}=0.008$, at 60 minutes of reperfusion: $\mathrm{P}=0.004)$. In the MP group and in the PP group also, CEFR gradually decreased during reperfusion periods and was lower than the baseline and/or after pretreatment values (P $<0.001$ for the MP group and the PP group). The CEFR values in the MP group and in the PP group were numerically higher than those in the control group, and only the CEFR value at 5

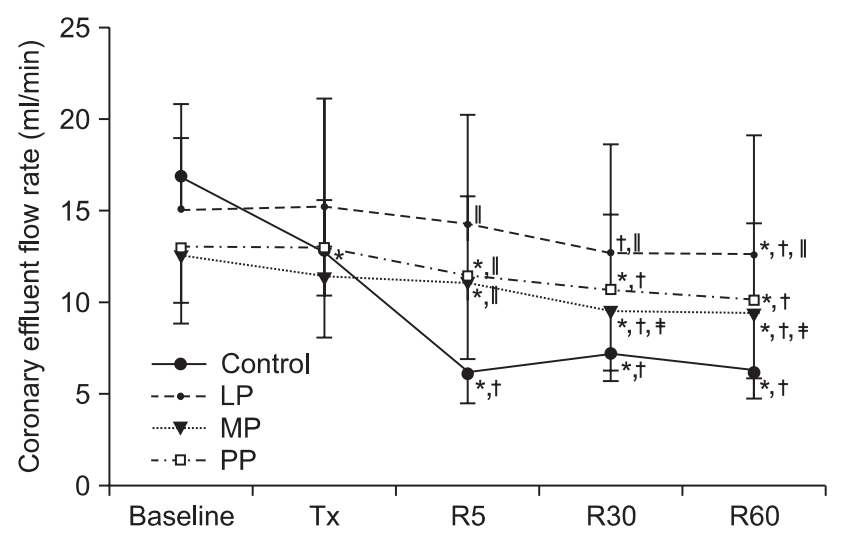

Fig. 6. Coronary effluent flow rate. Values are expressed as mean \pm SD. Control group: no treatment during pre-ischemic and reperfusion period, LP group: lipid emulsion propofol was infused during pre-ischemic and reperfusion period, MP group: microemulsion propofol was infused during pre-ischemic and reperfusion period, PP group: PP188 was infused during pre-ischemic and reperfusion period. Baseline: immediately after stabilization, Tx: pre-ischemic treatment, R5: at 5 minutes after reperfusion, R30: at 30 minutes after reperfusion, R60: at 60 minutes after reperfusion. Probability value was indicated as follows; ${ }^{*} \mathrm{P}<0.05$ vs. baseline, ${ }^{\dagger}$ vs. Tx, ${ }^{\ddagger}$ vs. R5, ${ }^{\S}$ vs. R30, "vs. control group, "vs. LP group, **vs. MP group. The coronary effluent flow rate was progressively decreased in all groups. However, in the LP group, the coronary effluent flow rate was significantly higher than that in the control group. minutes of reperfusion was statistically significant $(\mathrm{P}<0.001)$.

\section{Infarct size (Fig. 7)}

Infarct size was significantly smaller in the LP, MP, and PP groups $(14.27 \pm 2.41 \%, 15.77 \pm 2.40 \%, 14.40 \pm 1.97 \%$, respectively) than in the control group $(18.71 \pm 2.42 \%, \mathrm{P}<0.001)$.

\section{Discussion}

In the current study, we examined the influence of MP on myocardial injury resulting from ischemia and reperfusion in an isolated rat heart model and compared the myocardial protective effects between MP and LP. From a hemodynamic point of view, LP and MP produced similar results except for transient differences in LVESP and HR. In the MP group, LVESP was higher and HR was lower than that in the LP group during reperfusion. Moreover, infarct size did not differ significantly between the MP group and the LP group, and there seemed to be little difference between LP and MP in their myocardial protective effects against ischemia-reperfusion injury.

Within the first few minutes of reperfusion after myocardial ischemia, bursts of oxygen free radicals are generated [15], and these have been implicated as a cause of deleterious effects on cellular membrane proteins that maintain ionic homeostasis [16]. The beneficial effect of LP lies on its free radical scavenging properties during myocardial ischemia and reperfusion [2]. In addition, LP acts as a calcium antagonist preventing

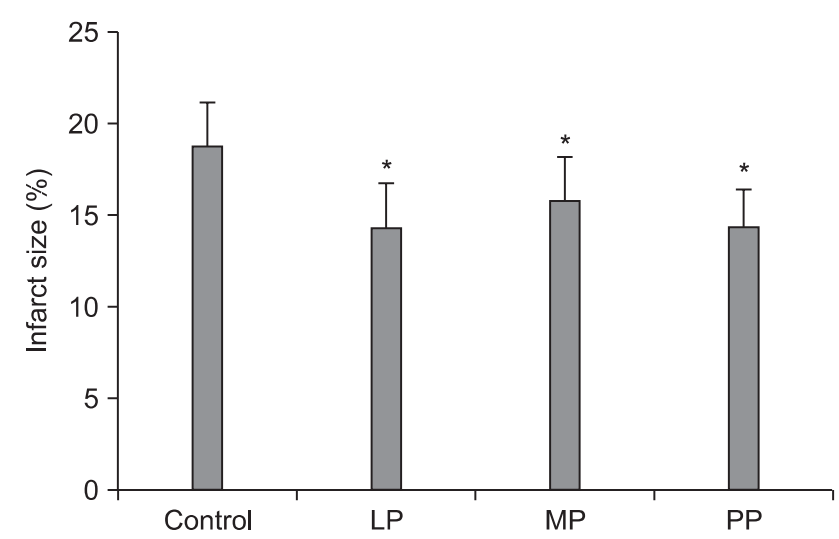

Fig. 7. Infarct sizes were calculated by using triphenyl tetrazolium staining method in all groups. Values are expressed as mean \pm SD. Control group: no treatment during pre-ischemic and reperfusion period, LP group: lipid emulsion propofol was infused during pre-ischemic and reperfusion period, MP group: microemulsion propofol was infused during pre-ischemic and reperfusion period, PP group: PP188 was infused during pre-ischemic and reperfusion period. Probability value was indicated as follows; ${ }^{*} \mathrm{P}<0.05$ vs control group. In the LP, MP and PP groups, the infarct size was significantly smaller than that in the control group. There were no significant differences in the infarct size between these three groups. 
intracellular calcium overload which causes myocardial injury [3,17-19]. The cardioprotective effects of PP188, an emulsifying agent in MP were also evaluated. Animal models underwent 2 hours of cardiopulmonary bypass with or without pretreatment with PP188. PP188 was associated with potentially beneficial changes in membrane protein expression, reduced capillary leakage, less hemodilution [7], and reduced membrane injury from ischemia and reperfusion $[8,11]$.

In many previous studies, propofol and PP188 have been demonstrated to have myocardial protective effects, but to the best of our knowledge there is no report about cardioprotection by MP which consists of propofol and PP188. In our study, we evaluated whether propofol emulsified with PP188, MP, had an additive or synergistic cardioprotective effect against ischemiareperfusion injury. LP is known to have myocardial protective effect in the concentration range $25-100 \mu \mathrm{M}(4.46-17.83 \mu \mathrm{g} /$ $\mathrm{ml}$ ) in isolated rat hearts $[1,2,12]$. Clinically, the recommended propofol concentration is $3-6 \mu \mathrm{g} / \mathrm{ml}$ for general anesthesia. We chose a LP concentration of $6 \mu \mathrm{g} / \mathrm{ml}$, this concentration has been clinically proven to be effective in myocardial protection against ischemia and reperfusion injury and falls in the recommended propofol concentration range. Protein binding of propofol ranges from 97 to $99 \%$ [20], and only a small fraction of administrated propofol exists as free propofol. However, not only free propofol in plasma but also propofol which is bound to protein or incorporated in cellular membrane can be effective in myocardial protection [1]. These findings mean that the total LP concentration in the perfusate solution, and not the free propofol concentration, is related to myocardial protection. The pharmacokinetics and pharmacodynamics of MP were similar to those of LP [14,21], and an MP concentration of $6 \mu \mathrm{g} / \mathrm{ml}$ was also used in the present study.

To compare infarct size in each group, we decided to use ischemic and reperfusion periods of 30 and 60 minutes, respectively. The ischemic and reperfusion periods were determined based on the previous studies. Infarct size reached its maximum after 35 minutes of global ischemia [22] and reperfusion for 60 minutes was optimal because the infarct appeared homogeneous at that time and did not become larger with longer reperfusion periods [23].

In the MP group, LVESP remains constant after initial stabilization but the heart rate was significantly reduced at 5, 30 and 60 minutes of reperfusion. In the PP group, LVESP was maintained near baseline unlike that in the LP group, and HR recovered towards the baseline value after reperfusion. Constant LVESP in the MP group may enable other vital organs to maintain blood flow even after ischemia and reperfusion injury and the low heart rate may help reduce myocardial oxygen demand and subsequent recovery of the injured myocardium.
LVEDP in the LP group and in the MP group was significantly lower than that in the control group during early reperfusion period. The PP group showed increased LVEDP similar to that in the control group. These findings implicate that propofol decreased LVEDP, which was increased due to ischemiainduced ventricular wall stiffness. This result is consistent with the previously reported propofol-induced myocardial protective effects [1]. In the MP group also, the LVEDP values were slightly higher than those in the LP group during reperfusion, but there were no statistically significant differences in LVEDP between the two groups. In all groups, LVEDP during the later reperfusion period was decreased towards the baseline values, and hence the long-term hemodynamic impact of PP188 could be negligible.

Infarct size in the LP, MP, and PP groups was smaller than that in the control group and infarct size did not differ between these 3 groups. Myocardial tissue edema can develop due to various pathologic conditions including myocardial ischemia, cardiopulmonary bypass, hypertension, and sepsis. Microvascular fluid loss is one of the leading causes of tissue edema, which contributes to cardiac dysfunction [24]. This damage to microvascular integrity is associated with loss of glycocalyx, reduction in the number of negatively charged molecules at the endothelial cell surface $[25,26]$. PP188 has membrane protecting effects by a sealing these damaged cell membranes [27]. This characteristic property of PP188 could produce myocardial protective effect against ischemia-reperfusion injury. According to our results, the infarct size in the MP group was similar to that in the LP group, even though MP consists of propofol and PP188. This finding demonstrated that PP188 did not interfere with the myocardial protective effects of propofol but it also did not have an additive or synergistic effect with propofol.

In terms of hemodynamics in this study, in the MP group, LVESP was maintained near baseline during the reperfusion period. But in the LP group, LVESP was lower than the baseline value during reperfusion. In the MP group, LVEDP remained close to the baseline during reperfusion unlike that in the LP group. These features are probably due to the nature of the emulsifier used in each propofol. Lipids are known to have no beneficial effects on myocardial protection from ischemia and reperfusion injury [28]. On the contrary, PP188 is known to have myocardial protective effects due to its cell membrane protecting effects [7-11]. However, the comparison between the MP group and the LP group revealed that hemodynamic parameters or infarct size were not significantly different between the two groups. In other words, the myocardial protective effect of MP was similar to that of LP, and this finding supported the conclusion that no additive cardioprotective effects were demonstrated with the use of MP.

In conclusion, we demonstrated that MP administered 
before and after myocardial ischemia results in hemodynamic changes similar to those with LP, and the concentrations of MP required for achieving myocardial protective effects following ischemia and reperfusion also did not differ from those of LP. PP188 used as an emulsifying agent for MP did not any influence the myocardial protective effect of propofol in spite of its own myocardial protective effects. We suggest that MP with less lipid-related side effects can be more useful than LP in anesthesia for cardiac surgery.

\section{References}

1. Ko SH, Yu CW, Lee SK, Choe H, Chung MJ, Kwak YG, et al. Propofol attenuates ischemia-reperfusion injury in the isolated rat heart. Anesth Analg 1997; 85: 719-24.

2. Kokita N, Hara A. Propofol attenuates hydrogen peroxide-induced mechanical and metabolic derangements in the isolated rat heart. Anesthesiology 1996; 84: 117-27.

3. Cook DJ, Housmans PR. Mechanism of the negative inotropic effect of propofol in isolated ferret ventricular myocardium. Anesthesiology 1994; 80: 859-71.

4. Devlin JW, Lau AK, Tanios MA. Propofol-associated hypertriglyceridemia and pancreatitis in the intensive care unit: an analysis of frequency and risk factors. Pharmacotherapy 2005; 25: 1348-52.

5. Park JW, Park ES, Chi SC, Kil HY, Lee KH. The effect of lidocaine on the globule size distribution of propofol emulsions. Anesth Analg 2003; 97: 769-71.

6. Bennett SN, McNeil MM, Bland LA, Arduino MJ, Villarino ME, Perrotta DM, et al. Postoperative infections traced to contamination of an intravenous anesthetic, propofol. N Engl J Med 1995; 333: 14754.

7. Egan JR, Butler TL, Cole AD, Abraham S, Murala JS, Baines D, et al. Myocardial membrane injury in pediatric cardiac surgery: An animal model. J Thorac Cardiovasc Surg 2009; 137: 1154-62.

8. Hunter RL, Luo AZ, Zhang R, Kozar RA, Moore FA. Poloxamer 188 inhibition of ischemia/reperfusion injury: evidence for a novel antiadhesive mechanism. Ann Clin Lab Sci 2010; 40: 115-25.

9. Watanabe M, Okada T. Lysophosphatidylcholine-induced myocardial damage is inhibited by pretreatment with poloxamer 188 in isolated rat heart. Mol Cell Biochem 2003; 248: 209-15.

10. Zhang R, Hunter RL, Gonzalez EA, Moore FA. Poloxamer 188 prolongs survival of hypotensive resuscitation and decreases vital tissue injury after full resuscitation. Shock 2009; 32: 442-50.

11. Justicz AG, Farnsworth WV, Soberman MS, Tuvlin MB, Bonner GD, Hunter RL, et al. Reduction of myocardial infarct size by poloxamer 188 and mannitol in a canine model. Am Heart J 1991; 122: 671-80.

12. Mathur S, Farhangkhgoee P, Karmazyn M. Cardioprotective effects of propofol and sevoflurane in ischemic and reperfused rat hearts: role of K(ATP) channels and interaction with the sodium-hydrogen exchange inhibitor HOE 642 (cariporide). Anesthesiology 1999; 91: 1349-60.
13. Baumert JH, Hein M, Gerets C, Baltus T, Hecker KE, Rossaint R. The effect of xenon on isoflurane protection against experimental myocardial infarction. J Cardiothorac Vasc Anesth 2009; 23: 614-8.

14. Jung JA, Choi BM, Cho SH, Choe SM, Ghim JL, Lee HM, et al. Effectiveness, safety, and pharmacokinetic and pharmacodynamic characteristics of microemulsion propofol in patients undergoing elective surgery under total intravenous anaesthesia. Br J Anaesth 2010; 104: 563-76.

15. Kloner RA, Przyklenk K, Whittaker P. Deleterious effects of oxygen radicals in ischemia/reperfusion. Resolved and unresolved issues. Circulation 1989; 80: 1115-27.

16. Goldhaber JI, Weiss JN. Oxygen free radicals and cardiac reperfusion abnormalities. Hypertension 1992; 20: 118-27.

17. Tadokoro H, Miyazaki A, Satomura K, Ryden L, Kaul S, Kar S, et al. Infarct size reduction with coronary venous retroinfusion of diltiazem in the acute occlusion/reperfusion porcine heart model. J Cardiovasc Pharmacol 1996; 28: 134-41.

18. Herzog WR, Vogel RA, Schlossberg ML, Edenbaum LR, Scott HJ, Serebruany VL. Short-term low dose intracoronary diltiazem administered at the onset of reperfusion reduces myocardial infarct size. Int J Cardiol 1997; 59: 21-7.

19. Wickley PJ, Shiga T, Murray PA, Damron DS. Propofol modulates $\mathrm{Na}^{+}-\mathrm{Ca}^{2+}$ exchange activity via activation of protein kinase $\mathrm{C}$ in diabetic cardiomyocytes. Anesthesiology 2007; 106: 302-11.

20. Schywalsky M, Ihmsen H, Knoll R, Schwilden H. Binding of propofol to human serum albumin. Arzneimittelforschung 2005; 55: 303-6.

21. Kim KM, Choi BM, Park SW, Lee SH, Christensen LV, Zhou J, et al. Pharmacokinetics and pharmacodynamics of propofol microemulsion and lipid emulsion after an intravenous bolus and variable rate infusion. Anesthesiology 2007; 106: 924-34.

22. Zacharowski K, Olbrich A, Otto M, Hafner G, Thiemermann C. Effects of the prostanoid EP3-receptor agonists M\&B 28767 and GR 63799X on infarct size caused by regional myocardial ischaemia in the anaesthetized rat. Br J Pharmacol 1999; 126: 849-58.

23. Schwarz ER, Somoano Y, Hale SL, Kloner RA. What is the required reperfusion period for assessment of myocardial infarct size using triphenyltetrazolium chloride staining in the rat? J Thromb Thrombolysis 2000; 10: 181-7.

24. Mehlhorn U, Geissler HJ, Laine GA, Allen SJ. Myocardial fluid balance. Eur J Cardiothorac Surg 2001; 20: 1220-30.

25. Chappell D, Dorfler N, Jacob M, Rehm M, Welsch U, Conzen P, et al. Glycocalyx protection reduces leukocyte adhesion after ischemia/ reperfusion. Shock 2010; 34: 133-9.

26. van den Berg BM, Vink H, Spaan JA. The endothelial glycocalyx protects against myocardial edema. Circ Res 2003; 92: 592-4.

27. Maskarinec SA, Hannig J, Lee RC, Lee KY. Direct observation of poloxamer 188 insertion into lipid monolayers. Biophys J 2002; 82: 1453-9.

28. Kobayashi I, Kokita N, Namiki A. Propofol attenuates ischaemiareperfusion injury in the rat heart in vivo. Eur J Anaesthesiol 2008; 25: 144-51. 